\title{
INTERACTION BETWEEN LECITHINS AND CHOLESTEROL AT THE AIR-WATER AND OIL-WATER INTERFACES
}

\author{
R. A. DEMEL \\ Laboratory of Organic Chemistry, State University of Utrecht, The Netherlands \\ P. JOOS \\ Laboratory of Physical Biochemistry, State University of Ghent, Belgium
}

Received 6 July 1967

\begin{abstract}
Spread mixed monolayers at the air-water of cholesterol and phospholipids (dipalmitoyl lecithin and stearoyl-oleoyl lecithin) were investigated using the Goodrich method. It was found that a weak interaction occurred between cholesterol and dipalmitoyl lecithin, on the contrary a large interaction was observed for the system cholesterol, stearoyl-oleoyl lecithin. Recently a theory was developed concerning the ideal behaviour of mixed adsorbed films. Since at the oil-water interface mixed adsorbed monolayers of cholesterol and the above mentioned phospholipids show an ideal behaviour, the conclusion was drawn that the interaction at the air-water interface is mainly due to Van der WaalsLondon attraction forces.
\end{abstract}

\section{Introduction}

Recently one of us ${ }^{1}$ ) took up again the work of De Bernard ${ }^{2}$ ) concerning the interaction of spread films of cholesterol and phospholipids at the airwater interface, spending much attention to the purity of the products used. It was found that the interaction between cholesterol and phospholipids greatly depends on the nature of the hydrocarbon chains of the phospholipids and it was put forward that this interaction was mainly due to Van der Waals-London attraction forces between the hydrophilic groups. This explanation is however not widely accepted ${ }^{3}$ ). Most experiments were hitherto performed at the air-water interface, where the Van der WaalsLondon attraction forces play a predominant part, at the oil-water interface however, the oil molecules penetrate between the hydrophilic moiety of the adsorbed or spread molecules, hence the Van der Waals-London attraction forces are annihilated. Unfortunately mixed films of cholesterol and phospholipids could not be spread as stable films at the oil-water interface in view of the solubility of the cholesterol in the "oil-phase". Recently, Joos ${ }^{4}$ ) and also Arcuri ${ }^{11}$ ) made a rigorous thermodynamical approach concerning the ideal behavior of adsorbed mixed films. The application of this theory to mixed films of cholesterol-phospholipid, adsorbed at the oil-water interface, 
should thus yield some indication about the nature of this interaction. Indeed, if the calculated values of the surface pressure as a function of the concentration of both components agree with the experimental ones, no interaction occurs, if however large discrepancies are found at the oil-water interface as for the system saponin-cholesterol ${ }^{12}$ ), the conclusion may be drawn that the interaction is not due to the Van der Waals-London attraction forces.

At first we reexamined the interaction at the air-water interface, between cholesterol and stearoyl-oleoyl lecithin and between cholesterol and dipalmitoyl lecithin, and an interaction was observed. In view of the just mentioned reasoning, this interaction was studied at the oil-water interface to point out if this interaction at the air-water interface, is due to Van der WaalsLondon attraction forces or not.

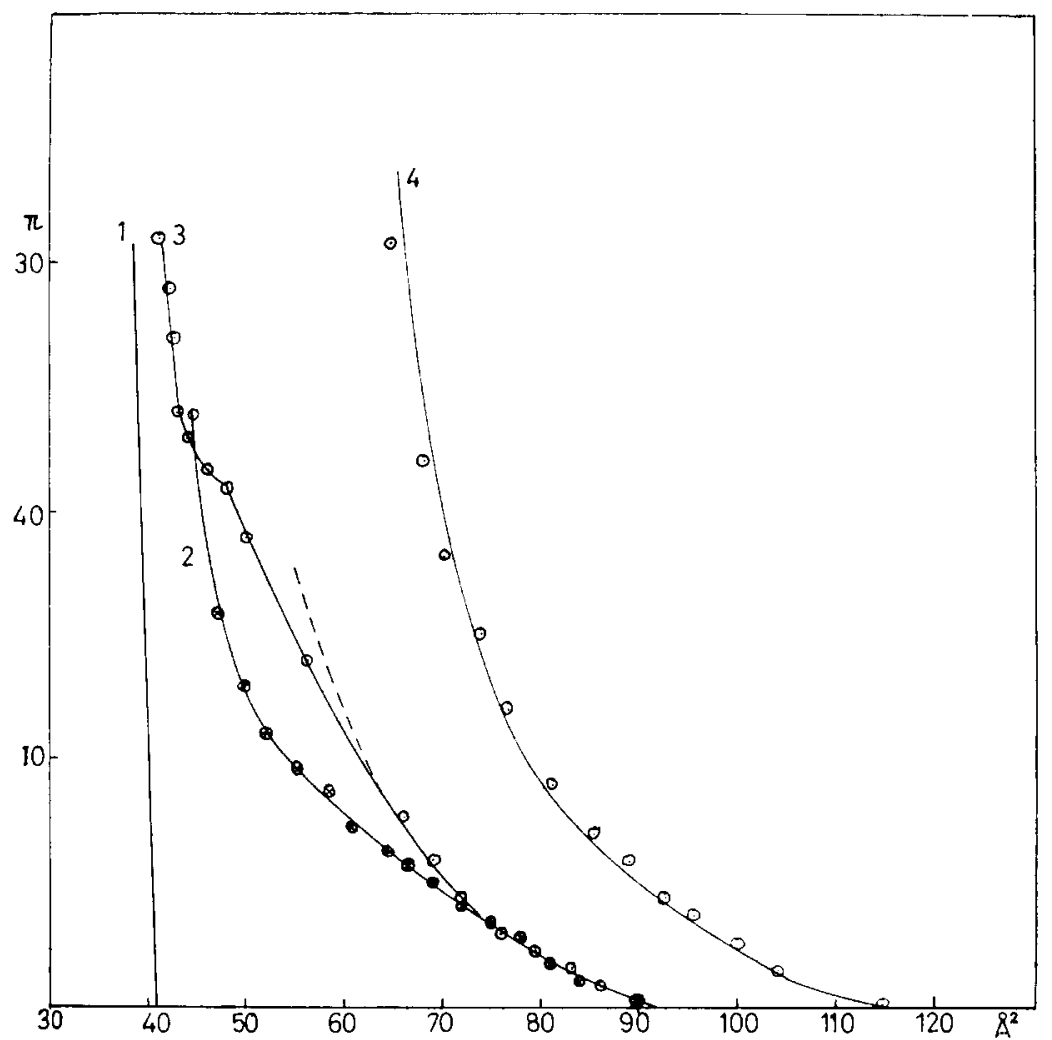

Fig. 1. Force-area $(\Pi-A)$ curves of cholesterol (1), dipalmitoyl lecithin (not purified 2, and purified 3) (dashed line according to eq. (2)) and stearoyl-oleoyl lecithin 4 (full line according to eq. (2)). 


\section{Materials and methods}

The stearoyl-oleoyl lecithin was a generous gift from the laboratory of Prof. L. L. M. Van Deenen. The dipalmitoyl lecithin was purchased from Sigma (grade II) and further purified by thin layer chromatography. From the difference of the force-area curves of the purified and unpurified product (see fig. 1), the great importance to perform such investigations with products of the highest purity is again emphasized. Cholesterol was purchased from Fluka (spreading experiments) and from U.C.B. (adsorption). Carbon tetrachloride and heptane were of analar grade and distilled to remove traces surface active impurities (e.g. fatts), $\mathrm{NaCl}$ was heated in a furnace at $\pm 600^{\circ} \mathrm{C}$ during several hours. The water was distilled twice in a pyrex apparatus.

For the spreading experiments a conventional Langmuir film balance was used. For the adsorption experiments at the oil-water interface the lecithin and cholesterol were dissolved in carbon tetrachloride or heptane. Interfacial tensions between water and carbon tetrachloride solutions were measured with a Wilhelmy plate of special design ${ }^{5}$ ), for the measurement of interfacial tensions between heptane solutions and water an ordinary Wilhelmy plate was used ${ }^{5}$ ). All interfacial tensions reported are equilibrium values (the solutions were allowed to stand during 2-3 hours).

\section{Results and discussion}

\section{Experiments at the air-water interface}

The force-area curves of cholesterol and the lecithins are given in fig. 1. The type of films of lecithins are liquid expanded. Hedge ${ }^{6}$ ) claimed that the $\Pi-A$ relationship of lecithin could be described by a Van der Waals equation:

$$
I=\frac{k T}{A-A_{0}}-\frac{a_{\mathrm{s}}}{A^{2}}
$$

using for $A_{0}$ (limiting area) a value of $40 \AA^{2}$. However our curves indicate that till a certain area $\left(115 \AA^{2}\right.$ for stearoyl oleoyl lecithin and $90 \AA^{2}$ for dipalmitoyl lecithin) the surface pressure is nearly zero, this fact is not explained by the Van der Waals equation using a constant value of $a_{\mathrm{s}}$ (Van der Waals constant). On the other hand Langmuir ${ }^{7}$ ) puts forward the following equation:

$$
\left(\Pi+\Pi_{\mathrm{c}}\right)\left(A-A_{0}\right)=k T
$$

where $\Pi_{\mathrm{c}}$, the cohesive pressure, is a constant, this in opposition to eq. (1) used by Hedge where $\Pi_{\mathrm{c}}=a_{\mathrm{s}} / A^{2}$.

Our curves are well described by eq. (2) till a surface pressure of 15-20 


\section{TABLE 1}

Parameters for lecithin monolayers (Langmuir equation).

Lecithin

Stearoyl-oleoyl lecithin

Dipalmitoyl lecithin

Lecithin of Hedge

$$
A_{0}\left(\AA^{2}\right)
$$

$\Pi_{\mathrm{c}}(\mathrm{dyne} / \mathrm{cm})$

dyne $/ \mathrm{cm}$, if the following appropriate values of $A_{0}$ and $\Pi_{\mathrm{c}}$ were used (table 1 ). Also the results of Hedge could be well described by eq. (2), taking a value for $A_{0}$ of $38 \AA^{2}$ instead of $40 \AA^{2}$.

It is also noteworthy that with dipalmitoyl lecithin a transition point in the force-area curve is found at $\Pi_{\mathrm{c}} \simeq 21 \mathrm{dyne} / \mathrm{cm}$ and $A \simeq 48.5 \AA^{2}$.

For a mixed spread film the force-area curve can be calculated from the force-area curves of the single component in the following way ${ }^{8}$ ): assume the surface, $S$, of a surface balance between the float and the barrier covered with two distinct monolayers of area $S_{1}$ and $S_{2}\left(S=S_{1}+S_{2}\right)$ containing $n_{1}$ and $n_{2}$ molecules of the two species respectively, separated by a thin thread in such a way that the surface pressure on both sides is equal. If this thin thread is removed, after mixing, the surface pressure will remain unaffected if no interaction occurs. A mean molecular area $(\bar{A})$ can now be defined:

$$
\begin{aligned}
& \bar{A}=\begin{array}{l}
S_{1}+S_{2} \\
n_{1}+n_{2}
\end{array}=\begin{array}{l}
n_{1} A_{1}+n_{2} A_{2} \\
n_{1}+n_{2}
\end{array} \\
& \left(A_{1}={ }_{n_{1}}^{S_{1}} ; A_{2}=\frac{S_{2}}{n_{2}}\right)
\end{aligned}
$$

From this relation the mean area $\bar{A}$ can be calculated for a given surface pressure $\Pi$.

In figs. 2 and 3 the results of mixed films of stearoyl-oleoyl lecithin and cholesterol are given. To avoid confusion only a few cases of the expected force-area curves (dotted lines) are given in fig. 2 . It is seen that a strong interaction between stearoyl-oleoyl lecithin and cholesterol occurs, confirming the previous results of Demel ${ }^{1}$ ).

Mixed films of dipalmitoyl lecithin and cholesterol show only a weak interaction especially at low pressures, as seen from fig. 4, while at higher pressures the interaction vanishes.

\section{Experiments at the oil-water interface}

It is well known that the surface (interfacial) pressure $(\Pi)$ concentration 


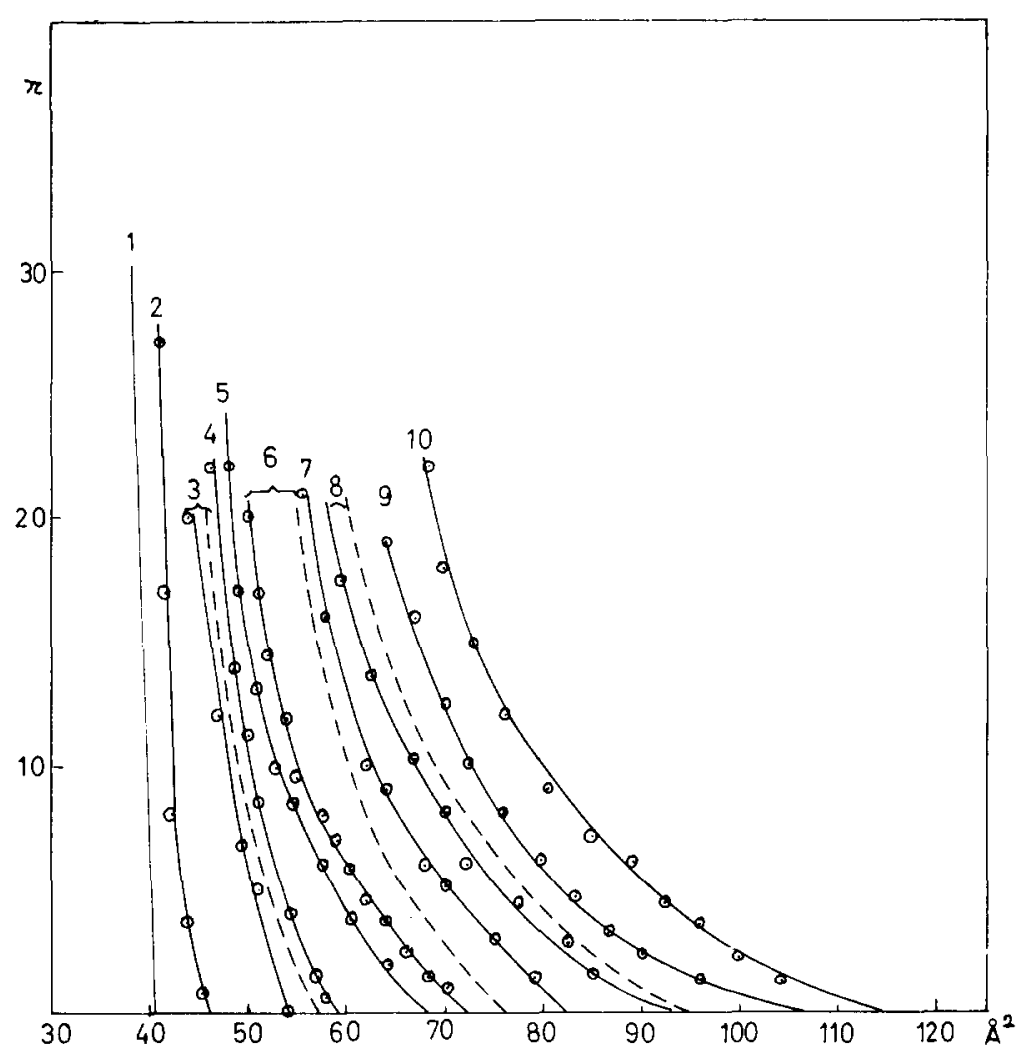

Fig. 2. Force-area $(\Pi-A)$ curves for mixed films of cholesterol and stearoyl-oleoyl lecithin. 1 : cholesterol; $2: x_{1}=0.07 ; 3: x_{1}=0.24 ; 4: x_{1}=0.32 ; 5: x_{1}=0.42 ; 6: x_{1}=0.53$; $7: x_{1}=0.63 ; 8: x_{1}=0.75 ; 9: x_{1}=0.87 ; 10: x_{1}=1$.

relationship for a solution containing a single surfactant can be described by the equation of von Szyszkowski ${ }^{9}$ ):

$$
\Pi=R T \Gamma^{\alpha} \ln \left(1+\begin{array}{c}
x \\
a^{\prime}
\end{array}\right)
$$

wherein: $\Gamma^{\infty}$ : the saturation adsorption,

$x$ : the molar fraction in the bulk ( $\simeq$ concentration),

$a^{\prime}$ : a constant related to the free enthalpy of adsorption.

This equation was obtained thermodynamically by Lucassen-Reynders and Van den Tempel ${ }^{10}$ ). Arcuri ${ }^{11}$ ) extended this equation for a system containing two or more surfactants, if the saturation adsorption values are the same, yielding:

$$
I=R T \Gamma^{\infty} \ln \left(1+\frac{x_{1}}{a_{1}^{\prime}}+\frac{x_{2}}{a_{2}^{\prime}}\right)
$$






Fig. 3. Variation of the mean molecular area as a function of the composition for mixed films of stearoyl-oleoyl lecithin and cholesterol at various surface pressures. $1: \Pi=5$ dyne $/ \mathrm{cm} ; 2: \Pi=10$ dyne $/ \mathrm{cm} ; 3: \Pi=15 \mathrm{dyne} / \mathrm{cm}$.

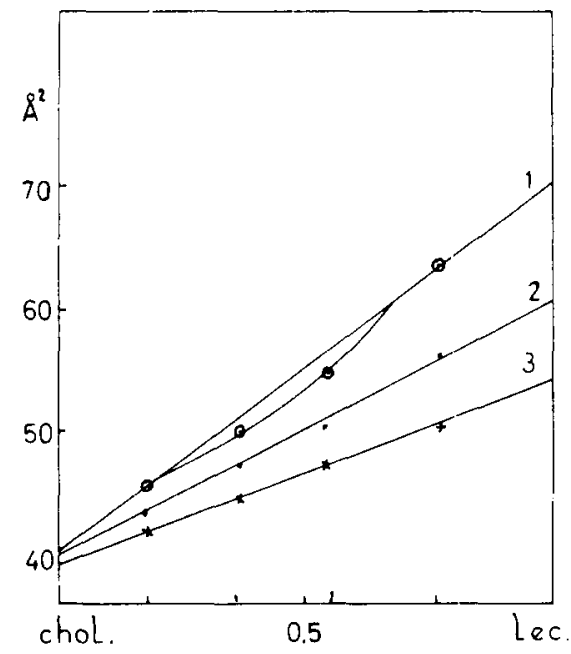

Fig. 4. Variation of the mean molecular area as a function of the composition for mixed films of dipalmitoyl lecithin and cholesterol at various surface pressures,

$1: \Pi=5$ dyne $/ \mathrm{cm} ; 2: \Pi=10 \mathrm{dyne} / \mathrm{cm} ; 3: \Pi=15 \mathrm{dyne} / \mathrm{cm}$. 
This equation was used for the system cholesterol-dipalmitoyl-lecithin ${ }^{12}$ ), since in this case the two saturation adsorption values are equal.

If however, as in the case cholesterol stearoyl-oleoyl lecithin the two saturation adsorption values are not equal, eq. (7) should be used. The von Szyszkowski equation was thermodynamically obtained for a special choice of the Gibbs dividing surface: $\Gamma^{\infty}=\Gamma_{0}+\Gamma_{1}\left(\Gamma_{0}\right.$ : adsorption of the water, $\Gamma_{1}$ : adsorption of the surface active component).

By the choice of the Gibbs dividing surface according to the monolayer model one obtains ${ }^{4}$ ):

$$
1=x_{0} \exp \left(\frac{-\Pi}{R T \Gamma_{0}^{\infty}}\right)+\frac{x_{1}}{a_{1}} \exp \left(\frac{-\Pi}{R T \Gamma_{1}^{\infty}}\right)
$$

wherein: $x_{0}$ : the molar fraction of the solvent in the bulk $(\simeq 1)$,

$\Gamma_{0}^{\infty}$ : the saturation adsorption of the solvent $\left(1.70 \times 10^{-9} \mathrm{~mol} / \mathrm{cm}^{2}\right)$,

$x_{1}$ : the molar fraction of the surfactant in the bulk,

$a_{1}:$ a constant,

$\Gamma_{1}^{\infty}$ : the saturation adsorption of the surfactant.

For a system containing two surfactants one obtains:

$$
1=x_{0} \exp \left(\frac{-\Pi}{R T \Gamma_{0}^{\infty}}\right)+{ }_{a_{1}}^{x_{1}} \exp \left(\frac{-\Pi}{R T \Gamma_{1}^{\infty}}\right)+\frac{x_{2}}{a_{2}} \exp \left(\frac{-\Pi}{R T \Gamma_{2}^{\infty}}\right)
$$

The values of the parameters: $a_{1}, a_{2}, \Gamma_{1}^{\infty}$ and $\Gamma_{2}^{\infty}$ are obtained from the surface pressure concentration relationship (eq. (6)) and summarized in table 2 for the different systems used.

The interfacial pressure concentration curves for the various components are given in figs. 5 and 6 .

The molar fraction at the interface could be calculated from the extended Langmuir equations ${ }^{12}$ ) (which are however inconsistent with thermodynamics):

$$
\Gamma_{1}=\frac{\Gamma_{1}^{\infty} C_{1} / a_{1}^{\prime}}{a_{1}^{\prime} a_{2}^{\prime}+a_{1}^{\prime} C_{2}+a_{2}^{\prime} C_{1}} ; \quad \Gamma_{2}=\frac{\Gamma_{2}^{\infty} C_{2} / a_{2}^{\prime}}{a_{1}^{\prime} a_{2}^{\prime}+a_{1}^{\prime} C_{2}+a_{2}^{\prime} C_{1}}
$$

TABLE 2

Parameters of the $\Pi$-conc. relationship.

\section{Component}

Cholesterol

Dipalmitoyl lecithin

Stearoyl-oleoyl lecithin

Cholesterol

Stearoyl-oleoyl lecithin

$\Gamma^{\infty}\left(10^{-9} \mathrm{~mol} / \mathrm{cm}^{2}\right)$

$a^{\prime}$

$a$

Remarks

0.44

0.44

0.30

0.44

0.30
$4.5 \times 10^{-4} \mathrm{~mol} / 1$

$2.5 \times 10^{-4} \mathrm{~g} / 1$

$1.3 \times 10^{-3} \quad \mathrm{~g} / 1$

$1.1 \times 10^{-4} \mathrm{~mol} / 1$

$4 \times 10^{-3} \mathrm{~g} / 1$
$1.110^{-3} \mathrm{~mol} / 1$ ) in

$-$

$\begin{array}{lll}4 & 10^{-3} & \mathrm{~g} / \mathrm{l}\end{array}$

$210^{-4} \mathrm{~g} / \mathrm{l}$ ) in

$\left.1.210^{-3} \mathrm{~g} / 1\right\}$ heptane 


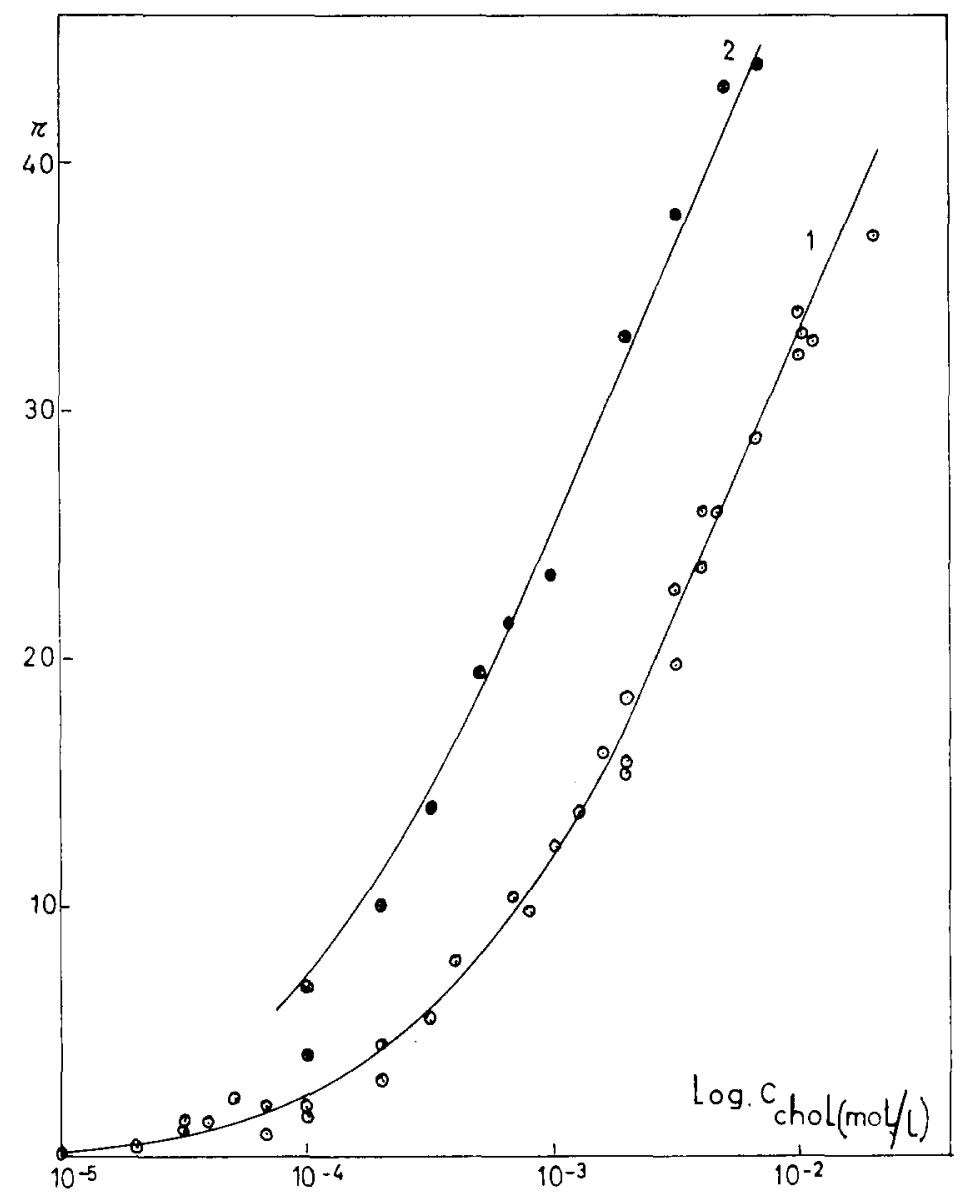

Fig. 5. Interfacial pressure/concentration relationship of cholesterol solutions in $\mathrm{CCl}_{4}$ and heptane against aqueous $\mathrm{NaCl}$ solutions. 1 : cholesterol in $\mathrm{CCl}_{4} / 0.1 \mathrm{~N} \mathrm{NaCl}$ and $1 \mathrm{~N} \mathrm{NaCl}$; 2: cholesterol in heptane/ $0.1 \mathrm{~N} \mathrm{NaCl}$ (solid lines: calculated curves).

The values of $\Pi$ from eq. (7) for mixed films were calculated by an electronic digital computer. Results are summarized in figs. 7, 8 and 9. For each run of experiments the ratio of the concentration of both components was kept constant, in this way the ratio of the adsorption of both components was constant too, this fact follows directly from eq. (8).

\section{Conclusions}

(1) Monolayers of dipalmitoyl lecithin and of stearoyl-oleoyl lecithin are of the liquid expand type. The $\Pi-A$ curves follow in a considerable range (up to 15 dyne $/ \mathrm{cm}$ ) eq. (2) (Langmuir). 


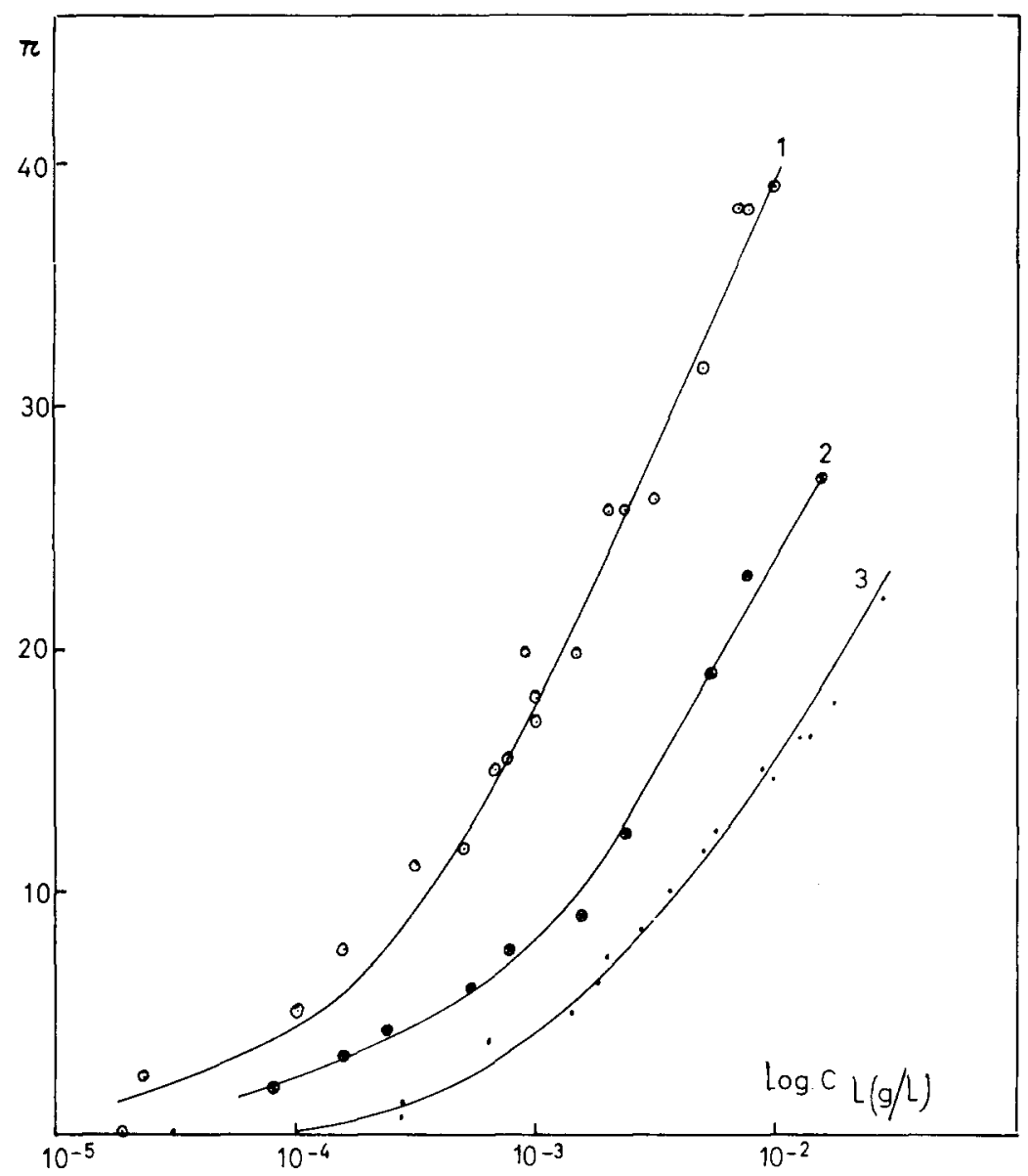

Fig. 6. Interfacial pressure-concentration relationship of lecithin solutions in $\mathrm{CCl}_{4}$ and heptane against aqueous $\mathrm{NaCl}$ solutions. 1: dipalmitoyl lecithin in $\mathrm{CCl}_{4} / 1 \mathrm{~N} \mathrm{NaCl}$;

2: stearoyl-oleoyl lecithin in heptane/0.1 N NaCl; 3: stearoyl-oleoyl lecithin in $\mathrm{CCl}_{4} / 0.1 \mathrm{~N} \mathrm{NaCl}$ (solid lines: calculated curves).

(2) A dipalmitoyl lecithin monolayer shows a transition point at a surface pressure of 21 dynes $\left(A=48.5 \AA^{2}\right)$. It appears also that a less pure sample of dipalmitoyl lecithin yields a completely different force area curve, and the transition point is not observed. In view of this it should be again emphasized to perform monolayers experiment with products of the highest purity.

(3) Mixed monolayers of cholesterol and dipalmitoyl lecithin shows only a weak interaction at low surface pressures $(5 \mathrm{dyne} / \mathrm{cm})$, at higher surface pressures this interaction vanishes. 


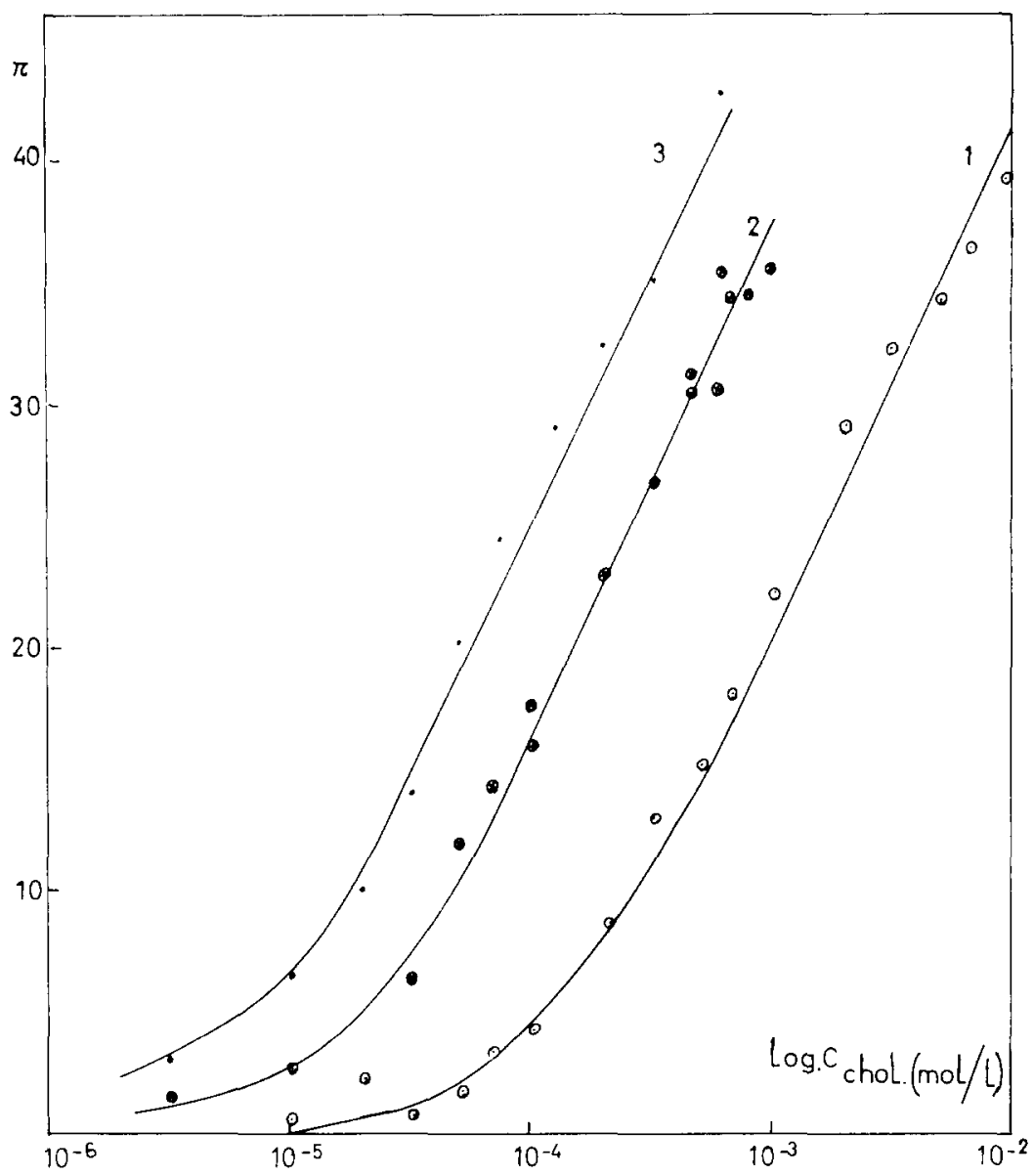

Fig. 7. Interfacial pressure-concentration relationship for mixed adsorbed films $\left(\mathrm{CCl}_{4}-0.1 \mathrm{~N} \mathrm{NaCl}\right.$ interface) of dipalmitoyl lecithin and cholesterol (solid lines: calculated curves) as a function of the concentration of cholesterol. $1: \Gamma_{1} \mathrm{e} / \Gamma_{\mathrm{rhol}}=50$; $2: \Gamma_{\text {led }} / \Gamma_{\text {ehol }}=16.1 ; 3: \Gamma_{\text {led }} / \Gamma_{\text {chol }}=1.61$.

(4) Mixed monolayers of cholesterol and stearoyl lecithin show a very marked interaction. The results are in agreement with the previous results of Demel $^{1}$ ).

(5) Adsorbed monolayers of cholesterol dipalmitoyl lecithin and stearoyl lecithin are ideal at the oil-water interface, since the equation of von Szyszkowski is followed. This equation was derived by Lucassen-Reynders and Van den Tempel ${ }^{10}$ ) on a purely thermodynamical basis.

(6) The interaction between cholesterol and phospholipids at the airwater interface results as a contraction effect. Mixed adsorbed films at the 


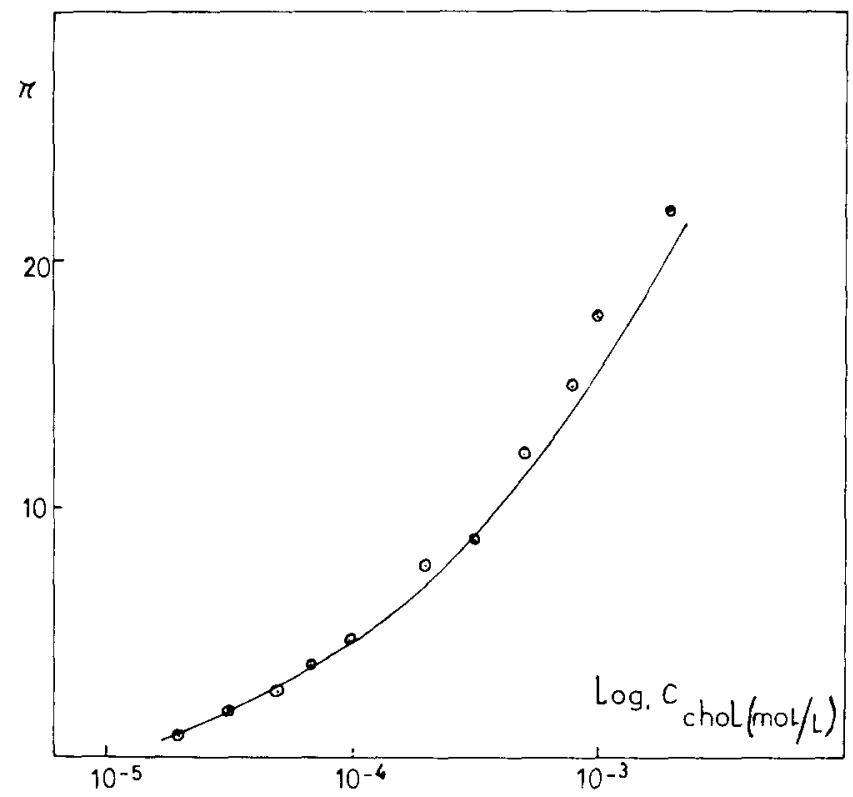

Fig. 8. Interfacial pressure-concentration relationship for mixed adsorbed films $\left(\mathrm{CCl}_{4}-0.1 \mathrm{~N} \mathrm{NaCl}\right.$ interface $)$ of stearoyl-oleoyl lecithin and cholesterol $\left(\Gamma_{\text {chol }} / \Gamma_{\text {lec }}=1.50\right)$ as a function of the concentration of cholesterol (solid lines: calculated curves).

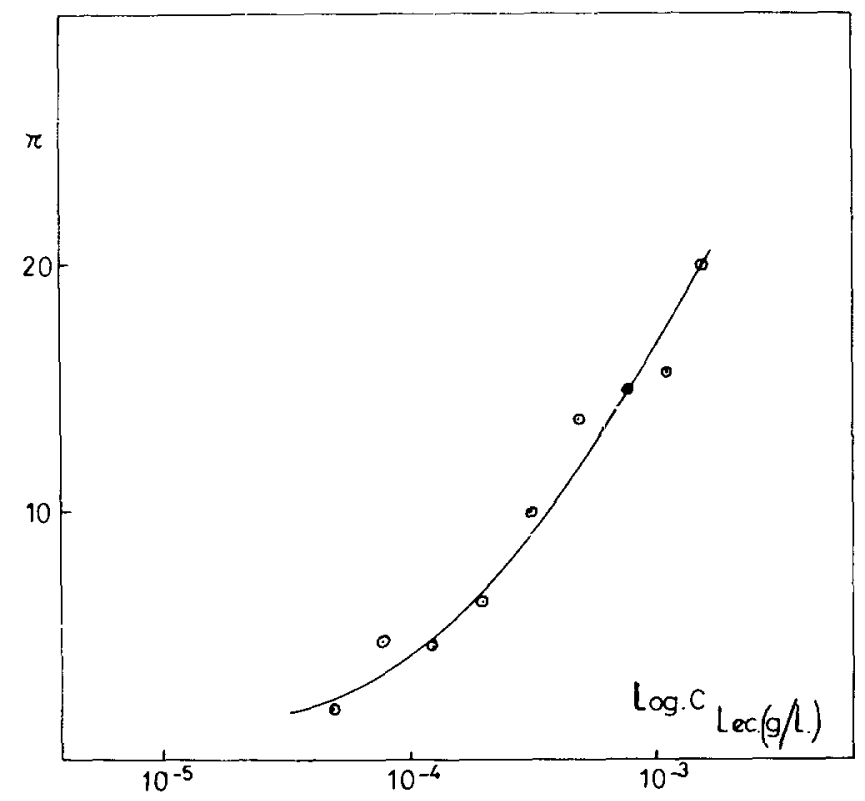

Fig. 9. Interfacial pressure-concentration relationship for mixed adsorbed films (heptane- $0.1 \mathrm{~N} \mathrm{NaCl}$ interface) of stearoyl-oleoyl lecithin and cholesterol $\left(\Gamma_{\mathrm{chol}} / \Gamma_{\text {lec }}=1\right)$ as a function of the concentration of lecithin (solid line: calculated curve). 
oil-water interface (carbon tetrachloride-water and heptane-water) show an ideal behaviour indicating that in this case no interaction occurs. In view of this one may conclude that the interaction observed at the air-water interface is mainly due to Van der Waals attraction forces, since at the oil-water interface this attraction forces are annihilated. Especially the system cholesterol-stearoyl-oleoyl lecithin is relevant. It is possible that at the airwater interface the lecithin and cholesterol molecules are arranged according to the models of Shah and Schulman ${ }^{3}$ ), but this Van der Waals attraction forces are the driving forces which promote this rearrangement.

It seems to us that the use of both methods (spread monolayer at the air-water interface and adsorbed monolayer at the oil-water interface) should give useful information about the nature of the interaction.

\section{Acknowledgements}

The authors are much indebted to Prof. L. L. M. Van Deenen for his interest and encouragement during the course of this work and for his kind gift of the lecithin samples. One of us (P. J.) is also indebted to Prof. R. Ruyssen for his interest in the course of this work, to the Rekenlaboratorium of Prof. C. Grosjean for the computation of the equations and to the Nationaal Fonds voor Wetenschappelijk Onderzoek for a travel grant.

\section{References}

1) R. A. Demel, L. L. M. van Deenen and B. A. Pethica, Biochim. Biophys. Acta 135 (1967) 11

2) L. de Bernard, Bull. Soc. Chim. Biol. 40 (1958) 164

3) D. O. Shah and J. H. Schulman, J. Lipid Res. 8 (1967) 227; B. A. Pethica, Surface activity and microbial cell. Soc. Chem. Ind., Monogr. 19 (1965) 85

4) P. Joos, in press

5) R. Ruyssen and R. Loos, Mededel. Koninkl. Vlaam. Acad. Wetenschap. Belg., Kl. Wetenschap. 2 (1945)

6) D. G. Hedge, J. Colloid Sci. 12 (1957) 417

7) J. T. Davies and E. K. Rideal, Interfacial phenomena. Academic Press, New York, (1961) p. 234

8) F. C. Goodrich, Proc. 2nd Intern. Congr. Surface activity, Vol. I (1957) p. 85

9) B. von Szyszkowski, Z. Phys. Chem. 64 (1908) 385

10) E. H. Lucassen Reynders and $M$. van den Tempel, preprints 4 th Intern. Congr. Surface activity, Brussels (1964)

11) C. Arcuri, Colloquium over grenslaagverschijnselen, Verhandel. Koninkl. Vlaam. Acad. Wetenschap. Belg., Kl. Wetenschap. (1966)

12) P. Joos, Mededel. Koninkl. Vlaam. Acad. wetenschap. Belg., Kl. Wetenschap. 3 (1966) 